\title{
Aa. Vv., «Parade Sauvage», Revue d'Études rimbaldiennes
}

\section{Mario Richter}

\section{Q OpenEdition}

1 Journals

\section{Edizione digitale}

URL: http://journals.openedition.org/studifrancesi/8330

DOI: 10.4000/studifrancesi.8330

ISSN: 2421-5856

\section{Editore}

Rosenberg \& Sellier

\section{Edizione cartacea}

Data di pubblicazione: 1 mai 2009

Paginazione: 199-200

ISSN: 0039-2944

\section{Notizia bibliografica digitale}

Mario Richter, «Aa. VV., «Parade Sauvage», Revue d'Études rimbaldiennes», Studi Francesi [Online], 157 (LIII | I) | 2009, online dal 30 novembre 2015, consultato il 11 janvier 2021. URL: http://

journals.openedition.org/studifrancesi/8330 ; DOI: https://doi.org/10.4000/studifrancesi.8330

Questo documento è stato generato automaticamente il 11 janvier 2021.

\section{(c) (i) $\odot$}

Studi Francesi è distribuita con Licenza Creative Commons Attribuzione - Non commerciale - Non opere derivate 4.0 Internazionale. 


\title{
Aa. Vv., «Parade Sauvage», Revue d'Études rimbaldiennes
}

\author{
Mario Richter
}

\section{NOTIZIA}

«Parade Sauvage», Revue d'Études rimbaldiennes, 21 novembre 2006, pp. 348.

Questo numero della nuova serie della rivista «Parade Sauvage» dedicata a Rimbaud e coordinata da Yann FRÉMY e Seth WHIDDEN contiene 24 contributi dovuti ad alcuni fra i migliori specialisti del poeta di Charleville (Jean-Pierre Babillot, Candice Nicolas, Alain Chevrier, David Ducoffre, Jacques Bienvenu, Antoine Fongaro, Seth Whidden, Michel Arouimi, Yann Frémy, Frédéric Canovas, Pierre Brunel, Ines Horchani, Frédérique Toudoire-Surlapierre, Zbigniew Naliwajek, Nicole Asquith, Jean-Louis Debauve, Pierre Borel, Jean Duché, Geneviève Hodin, Paul Claes, Christophe Bataillé, Marie-Joséphine Whitaker). Suddiviso in quattro sezioni (Études, Documents, Singularités, Comptes rendus), il fascicolo offre una panoramica critico-documentaria di notevole ricchezza e varietà. Segnaliamo in particolare una nuova articolata lettura del Bateau ivre (David DUCOFFre, Trajectoire du Bateau ivre, pp. 28-65), nella quale l'A. Si è impegnato a dimostrare che «Rimbaud a réussi le tour de force d'un poème historique dont la portée est universelle, tout en sachant que la finesse de sa compréhension est conditionnée par la surdétermination d'un événement précis: la Commune de 1871». Non meno interessante è l'articolo di Antoine FonGARo dedicato a Bannières de mai, che gli appare "peut-être l'expression la plus haute et la plus pure de l'aspiration à une poésie absolue où le poète disparaît en tant qu'individu» ("Bannières de mai": prélude, prologue, programme, pp. 93-111). Di particolare rilievo è lo studio che porta Michel ARouimI a valutare la considerevole incidenza di Edgar Poe su Rimbaud (Le goût de Rimbaud pour Edgar Poe, p. 124-147). Yann FRÉMY, l'apprezzato recente autore di una fondamentale thèse su Une saison en enfer (L'énergie poétique. "Une saison en enfer" d'Arthur Rimbaud, 2003) è tornato a studiare con perspicaci rilievi il controverso significato di Délires I, 
capitolo che gli appare «le lieu même de la stérilité des échanges» (Je est "une" autre. Lecture de "Délires I", pp. 148-166). Leggendo una volta di più l'“illumination" Aube mettendola a raffronto con À une passante di Baudelaire, Frédéric CANOVAS sostiene che il testo di Rimbaud «ne raconte pas l'échec du poète comme c'est le cas du sonnet de Baudelaire», ma che «il nous donne au contraire à voir 'un peu' de ce rêve du poète» (Imagerie et dynamique oniriques dans "Aube", pp. 167-180). Il vivace e interessante contributo di Pierre BRUNel (Rimbaud et Verlaine à Stuttgart, pp. 181-200), fra i vari e interessanti argomenti che affronta, tende a «retarder jusqu'au début du séjour à Stuttgart le travail accompli par Rimbaud sur ses poèmes en prose». Gli articoli che qui, per inevitabile brevità, non sono citati contribuiscono in vari modi a rendere questo volume degno del più grande pregio, particolarmente utile a chi voglia aggiornarsi circa l'attuale avanzamento delle ricerche su Rimbaud. Risulta a questo scopo di particolare profitto l'ampia, competente e articolata recensione che Bruno CLAISSE (pp. 305-315) ha dedicato al "livre-phare" di Steve Murphy Stratégies de Rimbaud (2004). 